\title{
La critique architecturale, objet de recherche
}

Architectural Criticism, an Object of Research

Hélène Jannière

\section{OpenEdition}

Journals

Édition électronique

URL : http://journals.openedition.org/crau/311

DOI : $10.4000 /$ crau.311

ISSN : 2547-5746

Éditeur

Éditions du patrimoine

Édition imprimée

Date de publication : 1 décembre 2009

Pagination : 121-140

ISBN : 978-2-85822-944-4

ISSN : 1296-4077

Référence électronique

Hélène Jannière, "La critique architecturale, objet de recherche », Les Cahiers de la recherche

architecturale et urbaine [En ligne], 24/25 | 2009, mis en ligne le 01 septembre 2017, consulté le 30 avril 2019. URL : http://journals.openedition.org/crau/311 ; DOI : 10.4000/crau.311 
Construire la critique en objet de recherche se heurte à des difficultés épistémologiques. Les conceptions de la critique varient selon que l'on considère la critique architecturale comme une des formes de critique des productions esthétiques, comparable à la critique des arts visuels, à la critique musicale, ou à l'inverse comme un discours interne au domaine de l'architecture, en relation avec I'histoire et les théories architecturales. L'analyse d'Hélène Jannière démontre avec rigueur que définir « la » critique architecturale de manière univoque relève d'une position doctrinale à priori : I'identification d'un objet de recherche ne doit-il pas se référer à des conjonctures particulières, qui ont façonné des configurations de la critique à des moments historiques et dans des milieux culturels déterminés?

\section{La critique architecturale, objet de recherche}

HÉ L ÈNE JANNIÈRE

Dénoncer la crise, voire annoncer la mort de la critique n'a rien d'inédit. Parmi les prises de position récentes, le recueil d'une centaine de chroniques du critique britannique Martin Pawley ${ }^{1}$, The Strange Death of Architectural Criticism (2007), réprouve l'abondance de papier glacé dans les revues d'architecture, en lieu et place d'une véritable critique. Si dans le domaine de l'architecture, Pawley est loin d'être le seul à s'étonner de la «la mort étrange de la critique », des constats analogues sont émis dans la sphère artistique. La disparition ou la crise de la critique d'art contemporaine sont imputées à l'inflation de l'exercice critique portant désormais sur n'importe quel objet, à la dilution de la critique dans des espaces médiatiques multiples et à la perte d'influence qui en résulte: "La critique d'art est dans une crise d'étendue mondiale. [...] Elle est en train de mourir, mais elle est partout ${ }^{2}$. " Ces mots ouvrent un récent pamphlet de l'historien d'art James Elkins, What happened to art criticism? (2003). Évoquant le trouble actuel de la critique d'art, ce titre qui sous-entend qu'auparavant ses objectifs, ses formes et sa fonction sociale étaient clairement définis, est concevable dans le domaine des arts, où la critique a constitué pendant deux siècles une pratique assez aisément identifiable par sa

1. Martin Pawley, The Strange Death of Architectural Criticism. Martin Pawley Collected Writings, Londres, Blackdog Publishing, 2007. Ce livre reprend le titre d'un article paru le 2 juillet 1998 dans The Architect's Journal dont Pawley a été le rédacteur en chef adjoint, outre ses fonctions de critique à Architectural Design, The Observer, The Guardian, au RIBA Journal et à Building Magazine.
2. James Elkins, What happened to Art Criticism?, Chicago, Prickly Paradigm Press, 2003, p. 2. 
fonction sur le marché de l'art et par l'équivalence, aujourd'hui remise en question, entre critique et jugement. II n'en va pas de même pour la critique architecturale, dont la nature et les fonctions n'ont cessé d'être débattues depuis la fin du XIXe siècle.

Comme la critique d'art, la critique d'architecture peut recouvrir plusieurs activités et registres de discours (du commentaire de l'actualité à l'histoire ou à l'esthétique), et ce dans plusieurs sphères: de la sphère de débat public, à laquelle depuis le XVIII siècle $^{3}$ la critique est historiquement liée (par les journaux et les Salons), à des cercles professionnels restreints. Si la difficulté de cerner l'objet critique est commune au domaine de l'art et à celui de l'architecture, les architectes et critiques invoquent pour leur part la nature complexe et presque indéfinissable de la critique architecturale. Ils imputent à la nature spécifique de l'architecture - une pratique, une discipline aux versants multiples - la difficulté d'en tracer les frontières et d'en inventorier les modes d'écriture : prise dans des contextes économique, technologique, social et urbain, celle-ci ne se laisse pas comparer à la production artistique, où ces dimensions paraissent moins déterminantes. Cette spécificité est parfois invoquée pour justifier les difficultés épistémologiques des études sur la critique.

Définir la critique - c'est-à-dire tracer son périmètre, déterminer son objet (les techniques, le programme, la forme, les solutions constructives, ou bien d'autres aspects de l'architecture), et désigner ses tâches - figure parmi les motifs principaux des écrits des architectes ou des rédacteurs des revues sur la critique. Mais au-delà des témoignages des acteurs, au-delà des contributions qui relèvent de l'opinion ou de prises de position (affirmer ce que doit ou devrait être la critique architecturale), la recherche se heurte à des difficultés épistémologiques qui ne semblent pas encore levées. Depuis le début des années 1990, plusieurs colloques et quelques publications ${ }^{4}$ dénotent l'intérêt des chercheurs pour ce sujet. Or, ces études peinent pour le moment à construire l'objet " critique », et à établir les conditions d'une recherche réellement cumulative. En effet, de nombreux auteurs s'arrêtent sur la question préalable du périmètre de la critique. Tracer ce périmètre peut les conduire à établir des typologies, aussi variables que les points de vue disciplinaires (sociologique, historique ou théorique) adoptés ${ }^{5}$. On peut voir dans cette répétition un indice de la difficulté de la recherche sur la critique. La plupart des articles illustrent le flou sémantique qui entoure les termes «critique architecturale ». Cette incertitude ne concerne pas que les frontières de la critique avec les théories et l'histoire de l'architecture: les termes " critique architecturale» recouvrent tour à tour une profession (si l'on se réfère aux critiques et à leur activité), ensemble de pratiques sociales ${ }^{6}$, un discours "savant " sur l'architecture, ou encore, comme l'expose I'historien et critique Peter Collins (1920-1981) dans Architectural Judgement ${ }^{7}$ (1971), un processus de décision interne à la conception architecturale.

Constituer la critique en objet de recherche ne peut faire l'économie d'une clarification épistémologique. Aussi cet article décline-t-il différentes conceptions de la critique d'architecture, en repartant de sa double origine dans la philosophie et dans la critique des productions esthétiques. Ces définitions varient de surcroît selon que
3. Richard Wrigley, The Origins of French Art Criticism. From the Ancien Régime to the Restoration, Oxford, Clarendon Press, 1993.

4. "The Space of Criticism », Lotus International, n० 72, mai 1992; Le Visiteur, n० 1 , automne 1995 (reprend partiellement une série de conférences tenues à la Société française des Architectes au printemps 1991); colloque "Critical Tools», NeThca (K-U Leuven, université de Louvain-la-Neuve, École d'architecture de La Cambre),
Bruxelles, 4-5 avril 2003: actes à paraître. Parmi les articles épistémologiques sur la critique: Fulvio Irace, "La critica architettonica: note per un dialogo », Op. cit. $n^{\circ} 76$, septembre 1989, pp. 10-23; Pattabi G. Raman, Richard Coyne, «The Production of Architectural Criticism », Architectural Theory Review, vol. V, n॰1, avril 2000, pp. 83-103.

5. Frédéric Pousin, «Aperçus sur la critique architecturale», Espaces et sociétés, n 60-61, mars 1992, qui reprend en grande partie les classifications opérées par Peter Collins, «Philosophie de la critique architecturale ", Architecture Mouvement Continuité, n॰9, octobre 1968 (initialement publié dans AIA Journal, vol. XLIX, n 1, 1968, pp. 46-49). 

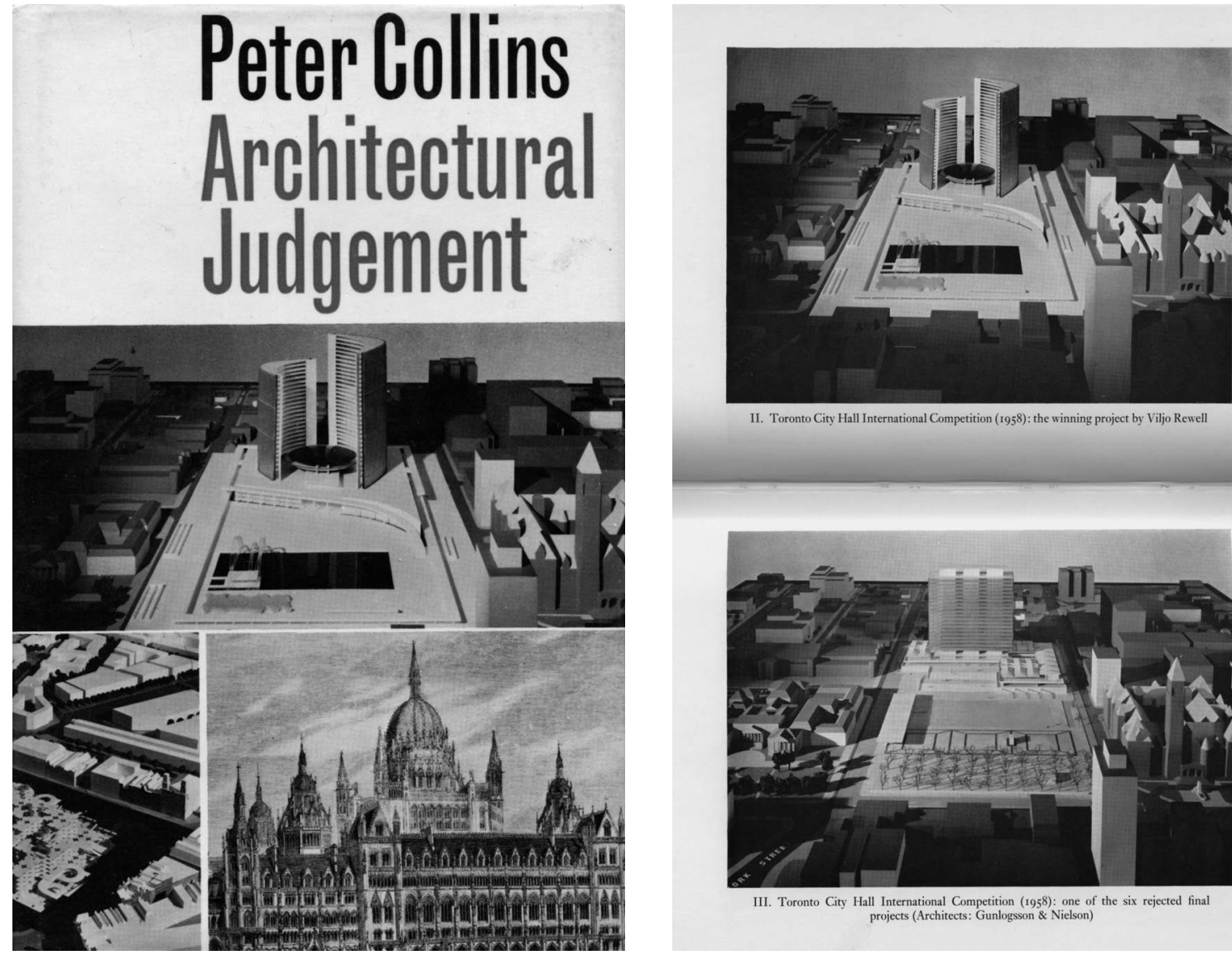

II. Toronto City Hall International Competition (1958): the winning project by Viljo Rewell

Peter Collins, Architectural

Judgement, Londres,

Faber \& Faber, 1971

Couverture et planches II

et III, Toronto City Hall

International Competition

(1958).

6. La tentation peut être grande d'étendre la critique à des pratiques de conception ou de négociation de l'objet architectural. L'ouvrage d'Agnès Deboulet, Rainier Hoddé et André Sauvage, La critique architecturale. Questions, frontières, desseins (Paris,

Éditions de La Villette, 2008), revendique une conception extensive de la critique, considérée comme un ensemble de pratiques sociales et professionnelles, allant jusqu'à englober des actions qui relèvent de l'enseignement (la correction), ou de la négociation entre maîtres d'ouvrage et concepteurs.

7. Peter Collins, Architectural Judgement, Londres, Faber \& Faber, 1971 
I'on considère la critique architecturale comme une des formes de critique, comparable à la critique des arts visuels ou à la critique musicale, ou à l'inverse comme un discours interne au domaine de l'architecture, voire à la discipline architecturale, en relation exclusive avec I'histoire et les théories, avec lesquelles elle ne cesse en outre de disputer ses frontières ${ }^{8}$. Auparavant, il met en perspective quelques éléments des débats sur la critique architecturale qui soulignent la diversité des significations de cette notion.

\section{La crise de la critique: historicité des débats Une vision instrumentale de la critique}

De nombreux écrits sur la critique émanant d'architectes ou de critiques professionnels constatent l'émergence de crises, engendrées tantôt par une crise des théories de l'architecture, tantôt par une crise de la profession ou encore par la disparition des avant-gardes et de l'engagement critique qui y était attaché. Depuis la fin du $X \mid X^{e}$ siècle, la presse architecturale condamne sporadiquement l'état insatisfaisant de la critique, son manque d'efficacité sur la qualité des édifices, ou va jusqu'à s'interroger sur son existence effective. Si elles ne sont pas aussi anciennes que les revues d'architecture (milieu du $\mathrm{XIX}$ siècle), de telles charges contre la critique sont présentes par exemple, dès les années 1890, dans Architectural Record, et perdurent tout au long du Xxe siècle. Une série d'articles dans la revue britannique The Architectural Review dans les années 1930, une autre dans I'américaine Pencil Points (ancêtre de Progressive Architecture) dans la décennie suivante, s'interrogent notamment sur la relation de la critique au " public ». La nature controversée de la critique architecturale, discours de spécialité touchant des cercles restreints de professionnels, de critiques, d'universitaires ou critique intermédiaire entre la profession et un public non spécialisé ${ }^{9}$, est en outre sans cesse débattue dans les revues d'architecture. C'est ainsi qu'en 1930 The Architectural Review s'élevait contre la critique issue des théories esthétiques et rappelait ce qui, de son point de vue, correspondait à sa tâche originelle, " conseillère sur le marché de l'art ${ }^{10}$ " - et qui vaut, par extension, pour la critique d'architecture, si l'on considère que celle-ci est un intermédiaire entre l'architecte et son client.

Plusieurs questions débattues depuis l'entre-deuxguerres dans les revues professionnelles présentent d'étonnantes permanences jusqu'à aujourd'hui : I'efficacité de la critique, ses critères, sa relation au public, son indépendance par rapport à la presse ou aux maîtres d'ouvrage, enfin son autonomie disciplinaire. Ces questions se rapportent surtout à la critique entendue de manière empirique comme commentaire de l'actualité, évaluation d'un édifice, à destination des professionnels ou d'un public plus large. Au-delà de telles considérations très empiriques, émises dans la première moitié $\mathrm{du} X \mathrm{x}^{\mathrm{e}}$ siècle, les problématiques de la critique architecturale - notamment ses relations avec les domaines de la théorie et de l'histoire - se sont depuis lors profondément transformées, comme en témoignent les débats sur la critique après 1945, rappelés brièvement ici.

\section{Après les combats de la critique engagée, la recherche de " critères objectifs"}

Dans l'après-guerre, on voit s'affirmer quelques éléments de réflexion sur la critique architecturale. Cette réflexion

\footnotetext{
8. Werner Szambien, «Incerte frontiere tra storia e critica dell'architettura », Casabella, n०547, juin 1988, p. 41-42.

9. Voir dans ce numéro la définition de la " critique publique » de Suzanne Stephens, critique à Architectural Record.

10. Eric Gill, "Painting and Criticism », Architectural Review, vol. LXVII, n 400, mars 1930, p. 111-112.

11. On assiste alors à une « prolifération de théories rivales qui se sont développées comme solutions à ce qui était unanimement ressenti comme l'absence d'une théorie adéquate pour la pratique et l'enseignement de l'architecture contemporaine ». Louis Martin, The Search for a Theory in Architecture: AngloAmerican Debates, 1957-1976, PhD Thesis, Princeton, NY, Princeton University, 2002, p. 9
}

12. L'Architecure d'aujourd'hui, vol. XXXIV, n॰116-117, novembre 1964: ce numéro présente quelques brèves prises de position de rédacteurs en chef de revues américaines (Thomas Creighton), de critiques $d^{\prime}$ architecture et d'art (Michel Ragon), de plusieurs architectes et ingénieurs et bien sûr des principaux critiques de la revue française (Julius Posener et Pierre Vago). 
s'engage alors que se profile une crise de l'héritage du Mouvement moderne: celle-ci s'accompagne d'une forte aspiration, par la jeune génération d'architectes et de critiques britanniques notamment, à la révision des fondements théoriques du Mouvement moderne et à l'établissement de nouvelles théories pour l'architecture de l'après-guerre, en réponse à ce qui est alors ressenti comme une lacune ${ }^{11}$. De 1945 à 1968, la presse professionnelle américaine et britannique s'est interrogée sur le rôle de la critique, son autonomie par rapport à la presse et aux architectes, ses instruments, ses critères d'évaluation ; tandis que dans les revues françaises, bien peu d'éléments filtrent de tels questionnements, si ce n'est dans un dossier, plutôt mince, de L'Architecture d'aujourd'hui en $1964^{12}$. Dans les périodiques américains et britanniques, les débats portent notamment sur l'évaluation esthétique de l'architecture, opposant les critères relevant du goût individuel, de la psychologie du critique et du plaisir esthétique, à des valeurs de portée plus générale, relatives à l'époque, la culture et l'environnement ${ }^{13}$. Par ailleurs, en 1956, James Marston Fitch salue le rôle important de la critique architecturale de l'entre-deux-guerres pour le succès du Mouvement moderne aux États-Unis, après la Seconde Guerre mondiale ${ }^{14}$.

Après la phase de diffusion du langage moderne et la reconnaissance du rôle prééminent d'une certaine critique, militante, dans cette domination doctrinale et stylistique, se développe une réflexion sur la nécessité d'objectiver et de partager les critères de la critique. Cette aspiration est alors partagée par de nombreux auteurs: notamment, pour Collins dans Changing Ideals in Architecture (1965), la recherche d'un système de valeurs « objectives » doit être au princeps de la critique architecturale et doit permettre de rompre avec la critique empirique et engagée de l'entre-deux-guerres, qui selon lui n'a réellement produit aucune véritable évaluation de l'architecture, afin de ne pas fragiliser les avant-gardes ${ }^{15}$. Dans la même optique, une série d'articles d'AlA Journal ${ }^{16}$ exprime en 1968 la nécessité d'évaluer non seulement l'architecture, mais aussi ses modes d'évaluation et leurs critères eux-mêmes.

Tenu à Cranbrook en 1964, le séminaire de l'American Institute of Architects, auquel participent Bruno Zevi, Peter Collins, Reyner Banham, Serge Chermayeff, Sybil Moholy-Nagy, Stephen W. Jacobs et Stanford Anderson, marque l'un des temps forts de la réflexion de l'aprèsguerre sur les rapports entre critique, théorie et histoire de l'architecture. La plupart des participants s'accordent sur le fait que c'est le jugement qui doit fonder la critique et la différencier de la théorie et de l'histoire. La question des critères du jugement anime, pour une part, les discussions de ce séminaire. Collins y dénonce la croyance, récente selon lui, selon laquelle il est possible de séparer totalement critique et histoire, critique et objectivité scientifique. L'idée selon laquelle l'émotion serait du côté de la critique, et l'objectivité scientifique du côté de I'histoire ou des sciences de l'art, est pour l'historien canadien un préjugé à combattre. Mais si, comme le voudrait Collins, la critique repose sur la base du jugement, elle ne peut être uniquement jugement de valeur. Il confère à la critique fondée sur la sensibilité personnelle de l'auteur une mention particulière : en référence à Oscar Wilde, il la qualifie de posture de "critique en artiste », dont il salue en John Ruskin et Vincent Scully les
13. N. E. Chotas, "The Critics' Role in Aesthetic Evaluation of Architecture ", Progressive Architecture, vol. XXXVIII, n॰ 7, juillet 1957, pp. 223-228.

14. James Marston Fitch, "The Shifting Bases of Contemporary Criticism », Progressive Architecture, vol. XXXVII, n॰ 6, juin 1956, p. 202. J. M. Fitch (1909-2000) a été rédacteur d'Architectural Record et Architectural Forum. Connu pour ses convictions en matière de défense du patrimoine, il a été l'initiateur des cours de conservation et de restauration à la School of Design, Planning and Preservation de Columbia University (NY).

15. P. Collins, Changing Ideals in Modern Architecture, Kingston/Montréal/McGill, Queen's University Press, 1988 [1 $1^{\text {re }}$ éd. Londres, Faber and Faber, 1965], p. 262-263.
16. Voir Abel Joseph Diamond, «A Plea for Performance Standards », AIA Journal (American Institute of Architects), juillet 1968, p. 54-55. 
"plus fins » représentants. Il lui préfère la critique fondée sur un système de principes, formulés antérieurement à l'édification du bâtiment: sur un tel système, la critique doit ainsi entretenir un lien étroit avec les théories. Si Collins appelle de ses vœux une critique normative, étayée par une théorie de l'architecture moderne (Changing Ideals in Architecture), d'autres auteurs souhaitent construire la critique sur un autre type de critères, "scientifiques » et parfois externes à l'architecture.

\section{Vers une critique "scientifique " ?}

Dans les années 1960, deux phénomènes ont, en effet, principalement marqué l'élaboration de nouvelles théories architecturales: d'une part la recherche, dans les méthodologies de la conception et les design studies (Christopher Alexander), d'une théorie "scientifique " de la forme et de la conception architecturales et, d'autre part, l'introduction de la linguistique et des différentes théories du signe, et l'accent mis sur les significations du langage architectural. Ces tentatives pour élaborer des instruments de lecture de l'architecture considérée comme un langage s'inscrivaient dans le contexte de la domination des sciences du langage dans les sciences humaines, caractéristique de cette décennie. Ces essais participaient également de la volonté diffuse de construire une théorie " universelle » apte à objectiver les questions de l'architecture et de son langage, auxquelles la sémiotique aurait apporté une validité. En 1974 (date du Congrès de Milan sur la sémiotique), la revue suisse Archithèse entrevoyait dans la sémiotique architecturale une possibilité de renouveler la critique sur de véritables critères ${ }^{17}$.
La sémiotique fut alors envisagée comme moyen de résoudre la crise du "dialogue » de la critique avec le public: elle serait à même d'offrir un mode de lecture et d'interprétation apte à décrypter les théories architecturales d'ordinaire peu accessibles à un large public. D'un point de vue heuristique, s'inscrivant dans ce mouvement d'introduction des sciences du langage dans les analyses de l'architecture, plusieurs ouvrages ont eu l'ambition de décrypter le lexique, les instruments et les rhétoriques de la critique. Architecture and its Interpretation (1979) ${ }^{18}$ de Juan Pablo Bonta s'est intéressé, à partir d'une analyse des discours critiques, à la formation et à la " cristallisation » des interprétations canoniques de certains édifices de l'entre-deux-guerres, encourageant indirectement une toute neuve génération de recherches sur I'historiographie du Mouvement moderne. Moins connu, Architecture and Critical Imagination de Wayne Attoe (1978) constitue une tentative de classification des rhétoriques de la critique architecturale et reste, trente ans après sa parution, l'une des rares études épistémologiques sur ce sujet ${ }^{19}$. II tente d'en identifier les modèles (critique littéraire ou critique d'art) et dessine une typologie, distinguant trois grandes postures de critique: normative (qui juge à l'aune d'un ensemble de critères préétablis), interprétative (la plus subjective, exprimant la sensibilité du critique) et enfin descriptive (la plus factuelle, décrivant les matériaux, la construction, les formes). Pour chacune d'elles, l'auteur énumère les approches possibles: la critique normative par exemple peut se fonder soit sur une doctrine, soit sur un système plus complexe de critères, soit sur la notion de type architectural, ou encore la notion de mesure.
17. «Dans le domaine de la littérature et de l'art, il existe depuis deux cents ans au moins des formes de critique qui essaient d'établir un dialogue entre producteurs et consommateurs. Actuellement ce phénomène ne se vérifie plus dans le secteur de l'architecture. [...] II se peut que la sémiologie de l'architecture soit capable de dépasser cette situation où la théorie n'est accessible qu'aux professionnels de la construction. Ceci dans la mesure où elle s'occupe de la perception et de l'expérience et non seulement de l'utilisation de l'environnement. » Antonio Hernández, "Zur Geschichte der Architekturkritik », Archithèse, no 10, Zurich, 1974, p. 2.

18. Il est parmi les premiers à analyser les récits historiques et critiques sur l'architecture du Mouvement moderne.

19. Citons également, plus récemment: Pattabi G. Raman, Richard Coyne, art. cit. note 4 .
20. Voir Hélène Jannière, « La critique architecturale à la recherche de ses instruments: L'Architecture d'aujourd'hui et Architecture Mouvement Continuité, 1967-1980", in Alexis Sornin, Hélène Jannière, France Vanlaethem (dir.), Les revues d'architecture dans les années 1960 et 1970/Architectural Periodicals in the 1960s and 1970s, Montréal, CCA, IRHA, pp. 269-292. 
Parallèlement à la recherche d'une théorie « scientifique » pour l'architecture, tant dans les sciences du langage que dans celles de la conception, d'autres instruments ont été convoqués dans la critique architecturale. Dans les années 1960 et surtout 1970, celle-ci porte les marques de l'introduction des sciences humaines et sociales dans les analyses de l'architecture, qui acquièrent ainsi une dimension scientifique. Les analyses marxistes de l'espace urbain et architectural, les analyses linguistiques, anthropologiques, sociologiques de l'architecture sont ainsi transposées dans la critique ${ }^{20}$.

\section{Manfredo Tafuri, « Per una critica dell'ideologia architettonica ", Contropiano, $\mathrm{n}^{\circ}$ 1, 1969.}

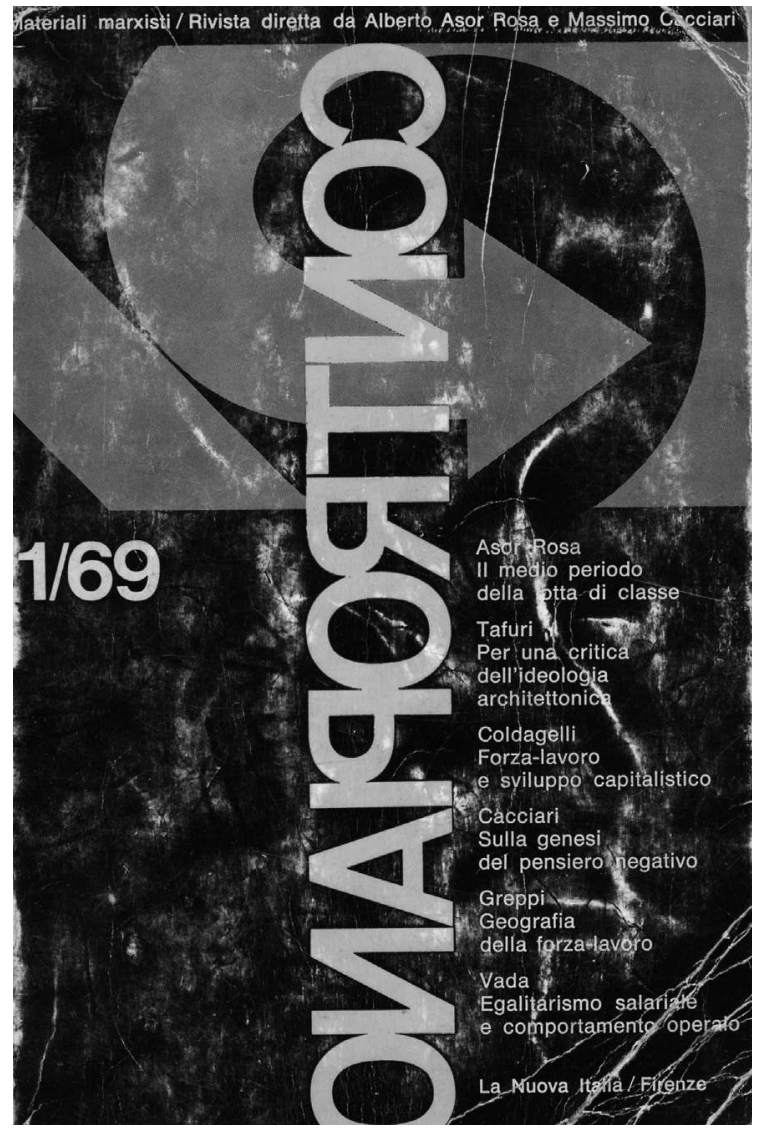

De la critique opératoire aux formes contemporaines de critique

Mais outre ces tentatives successives, I'une des ruptures importantes dans I'histoire de la critique architecturale est marquée par la fin du "projet moderne », et par conséquent le déclin de la critique engagée ou opératoire qui en avait accompagné les développements jusque dans les années 1960. Dans Théories et histoire de l'architecture (1968), Manfredo Tafuri (1935-1994) a, le premier, défini cette forme de critique et l'a condamnée comme " analyse de l'architecture (ou des arts en général), qui aurait pour objet non un relevé abstrait mais la "projétation" d'une direction poétique précise, anticipée dans ses structures et que révèlent des analyses historiques programmées et déformantes ${ }^{21} »$. Depuis, la rupture avec la critique opératoire a été saluée comme une transformation positive: son abandon permettrait enfin de développer la critique sur de véritables critères «scientifiques » universels (c'est l'ambition des années 1960 et 1970). Plus récemment, la critique opératoire est à l'inverse objet de nostalgie; ainsi, l'un des leitmotive des discours des critiques sur la critique est l'apologie de la critique engagée, qui représente un mode antérieur et idéalisé de critique architecturale. Les exemples les plus fréquemment cités sont la critique liée aux avant-gardes de l'entre-deux-guerres, ou la critique britannique de I'après-guerre (de The Architectural Review à Architectural Design, avec des critiques engagés comme Nikolaus Pevsner ou Reyner Banham). Aujourd'hui, certains critiques opposent ce modèle d'engagement à la critique "star system ${ }^{22}$ ", propre à la collusion entre critique et stratégies communication ${ }^{23}$.

21. Manfredo Tafuri, Théories et histoire de l'architecture, Paris, SADG, 1976, p. 189 [édition originale1968].

22. François Chaslin, "Critique d'architecture », in Dictionnaire de l'architecture du XXe siècle, Paris, Hazan, Institut français d'architecture, 1995, p. 223-224; Id., " Architecture and Criticism », in Mohammad al-Asad, Majd Musa (dir.), Architectural Criticism and Journalism: Global Perspectives, Turin, Allemandi, 2006, pp. 21-27. 
Depuis les années 1980, de nombreux observateurs soulignent le changement de paradigme de la critique architecturale. Face à des tendances architecturales de plus en plus individualisées, face à l'absence de doctrines dominantes et au délitement des idéologies, le rôle de la critique n'est-il pas plus de cartographier que de juger, d'évaluer, ce qui était sa fonction première? «Moins que des corpus de théories, ce à quoi nous sommes confrontés sont des situations de facto, des projets qui ont cherché à trouver leur cohérence dans la conjoncture spécifique de chaque événement. [...] Depuis une multiplicité de plates-formes, aujourd'hui la critique peut entreprendre la production de cartes ou de descriptions qui, comme des cartes topographiques, révèlent la complexité d'un territoire - sa forme résulte d'agents géologiques opérant en silence sur une masse immobile et néanmoins traversée de courants, de flots, de changements et d'interactions donnant naissance à d'incessantes mutations ${ }^{24}$. "Recensant pour la revue Casabella cet ouvrage, Diferencias, d'Ignasi de Solà Morales (1995), I'historien Carlo Olmo rapporte ces nouvelles « topographies de la critique " à la situation de l'architecture contemporaine et à l'impossibilité pour la critique de fonctionner sur le modèle précédent, celui de la critique de tendance, délimitant et nommant des courants et des mouvements ${ }^{25}$. Cette situation démontre plus encore l'impossibilité voire l'anachronisme de toute tentative normative (la critique comme jugement).

Enfin, I'une des tendances de ces dernières années est de discuter l'autonomie du texte critique par rapport à son (ses) référent(s) et à la discipline architecturale. Dans le récent Critical Architecture (2007) ${ }^{26}$, Jane Rendell propose, en lieu et place de critique d'architecture, le terme d'architecture writing. Dans ce type de discours sur l'architecture, la dimension littéraire et l'intérêt porté à la structure textuelle deviennent dominants, comme c'est le cas pour l'art writing qui selon cet auteur remplace, après la période postmoderne, la critique d'art au détriment des qualités polémiques et de débat de cette dernière $^{27}$.

\section{Genèse de deux conceptions de la critique}

Ce panorama, qui mériterait de plus amples approfondissements, montre toutefois qu'il est peu pertinent de se limiter à une seule conception de la critique : ses définitions dépendent des périodes, des champs disciplinaires et des contextes culturels. Les mots criticism, critica et critique recouvrent ainsi, des États-Unis à l'Italie et à la France, des notions différentes. La première est celle du commentaire critique indexé sur le présent, qui évalue et interprète une œuvre dans sa singularité : cette conception prévaut en France, où la critique recouvre à la fois une activité (parfois même une profession) et un genre de texte, avant de désigner un discours spéculatif sur l'architecture. Cette acception est très fortement empreinte des définitions canoniques de la critique d'art. À l'inverse, discours spéculatif sur l'architecture " en général ", la seconde acception est celle d'une critique qui ne s'attache pas à commenter une œuvre singulière, mais qui tend à recouvrir l'histoire critique, la théorie critique (notamment aux États-Unis) ou confine avec l'histoire (dans les conceptions italiennes). Aux marges de la critique, généralement considérée comme un discours, écrit ou non, on peut en outre mentionner une définition
23. Valérie Devillard, Architecture et communication. Les médiations architecturales dans les années 80 , Paris, Éditions Panthéon-Assas, LGDJ, 2000.

24. Ignasi de Solà Morales, Differences. Topographies of Contemporary Architecture, Cambridge (Mass.), The MIT Press, 1997, p. 16 [édition originale Diferencias. Topografía de la arquitectura contemporánea, Barcelone, Gustavo Gili, 1995].
25. Carlo Olmo, «Topografie della critica ", Casabella, n 629, décembre 1995, p. 32.

26. Jane Rendell, Jonathan Hill, Murray Fraser, Mark Dorrian, Critical Architecture, Londres, New York, Routledge, 2007.
27. L'auteur s'appuie sur une série d'articles dans la revue d'art britannique Art Monthly (2003), qui fait le constat d'un remplacement progressif de la critique d'art avec ses qualités polémiques, aiguës, contestataires par l'art writing. 
par « extension », conception qui s'appuie sur la fonction critique de l'œuvre - revendiquée en art comme en architecture. Dans Critica del discorso architettonico, I'architecte Vittorio Ùgo distinguait deux formes principales de la critique: "Interpréter de manière critique une œuvre architecturale peut avoir une signification double: celle de l'architecte qui la sélectionne et l'assume historiquement comme matériel pour donner forme à son propre projet [...] celle du critique qui en extrait au contraire un motif pour la production d'un "discours" : donc d'une œuvre nouvelle dont le statut diffère substantiellement de celui de l'œuvre qui l'a inspirée ${ }^{28}$. » Les architectes ou les théoriciens de l'architecture considèrent souvent la conception architecturale comme une forme de critique. Pour Vittorio Gregotti, le projet architectural est instrument de « critique historique et théorique », notamment à I'égard du Mouvement moderne ${ }^{29}$. De même, dans Architecture and Critical Imagination, Attoe rejette le modèle de la critique littéraire et celui de la critique d'art. II élargit ses instruments à ceux, graphiques, de l'architecte: « Je suis opposé à voir dans le critique seulement un personnage littéraire. On peut faire des distinctions, passer au crible, décrire, expliquer et interpréter dans un laboratoire ou un studio, et avec un appareil photographique ou un marqueur, aussi aisément et utilement qu'avec une machine à écrire. La critique devrait être envisagée en termes de tactique et d'intentions, et non en termes de média employé ${ }^{30}$. „ Cela rejoint, en partie, la position adoptée par Bruno Zevi dans les années 1960, lorsqu'il proposait de réfléchir à une critique empruntant les moyens propres à l'architecture, dessins et maquettes, au détriment de la critique écrite, inefficace selon lui pour les architectes. En outre, Attoe se réclame d'une approche extensive de la critique, ne la limitant ni aux articles grand public de la presse professionnelle, ni aux essais «épais et impopulaires » qui relèveraient de la critique «savante ${ }^{31}$ »: toute "réaction » selon lui, à l'environnement construit, doit être considérée comme une forme de critique ${ }^{32}$, ce qui inclut l'acte de concevoir, en architecture comme à l'échelle urbaine. Le récent débat américain sur la « criticality » ou potentialité critique de l'architecture, dont il sera question ici, en est un autre témoin.

\section{Critique et théorie, théorie critique}

« La critique architecturale n'est ni un genre littéraire, ni une profession. C'est, avant tout, une attitude intellectuelle par les moyens de laquelle le discours devient - dans la solitude et la conscience de la crise - jugement, séparation, décision ${ }^{33}$. "Par ces mots, Ignasi de Solà Morales rejette l'analogie de la critique architecturale avec la critique artistique entendue comme genre littéraire, définition forgée au début $d u x x^{e}$ siècle à partir de la pratique de la critique des Salons depuis le XVIII siècle. En déclarant que la critique architecturale n'est pas une profession, Solà Morales repousse l'idée de critique comme pratique sociale, et par conséquent la pertinence de l'aborder d'un point de vue sociologique ${ }^{34}$. Ainsi prévaut la volonté d'établir une définition de la critique d'architecture, non en repartant des conceptions canoniques de la critique d'art, mais en recourant à certaines des notions philosophiques du mot critique.

Si l'on remonte aux significations sédimentées du mot critique à travers les siècles, celui-ci renvoie non seulement à plusieurs conceptions, mais à plusieurs domaines
28. Vittorio Ugò, Kriteria. Critica del discorso architettonico, Milan, Guerini Studio, 1994 , p. 20

29. Vittorio Gregotti, « Necessità della teoria », Casabella, n 494, septembre 1983, réédité dans Questioni di architettura, Turin, Einaudi, 1986, p. 62.

30. Wayne Attoe, Architecture and Critical Imagination, Chichester, New York, Brisbane, Toronto, John Wiley and Sons, 1978, p. XVI.
31. Ibid.

32. Ibid., p. XIX.

33. I. de Solà-Morales, op. cit. note 24, p. 5.

34. Ce qu'ont fait avec succès plusieurs auteurs, dont Hélène Lipstadt: Pour une histoire sociale de la presse architecturale: la Revue Générale de l'Architecture et César Daly (1840-1888), thèse de doctorat de $3^{e}$ cycle, Paris, ÉHESS, 1979. 
d'application distincts, successivement apparus dans I'histoire, de I'Antiquité au XVIII siècle: la philologie - premier sens du mot critique, critique textuelle ou exégèse des textes, auquel est attachée l'acception du mot grec criticos (" celui qui s'occupe d'études philo$\left.\operatorname{logiques}^{35} »\right)-$, la critique historique (I'examen des sources), la philosophie, et la critique dans le domaine des productions esthétiques, littérature et $\operatorname{art}^{36}$. Dans les significations contemporaines du mot critique, la principale dualité se joue en effet entre la critique entendue comme activité philosophique et la critique dans le domaine des productions esthétiques - ces deux sens étant néanmoins imbriqués, la critique esthétique faisant toujours appel à la théorie (" Au-delà même du goût, la critique va de pair avec la théorie, autant que la théorie appelle toujours la critique $\left.{ }^{37} »\right)$. Ainsi, pour les spécialistes de l'esthétique: « La critique est à la fois l'opération interne à la philosophie qui, pour parler vite, vise à examiner les conditions de la connaissance, et le travail de réflexion, d'évaluation qu'assume un certain nombre

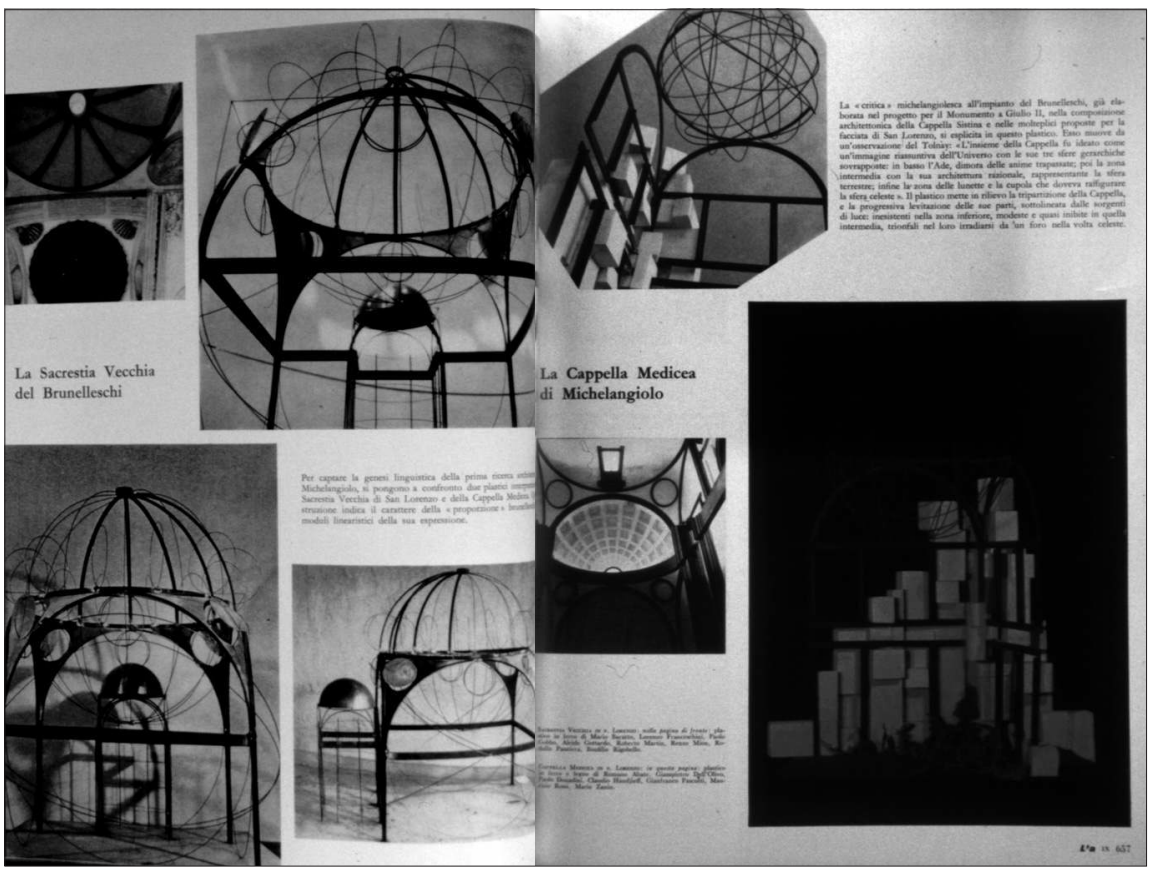

35. Claude Amey, "Une question critique », in Séminaire Interarts (1999-2000), L'œuvre d'art et la critique, Paris, Klincksieck, 2001, p. 17

36. Jean-René Ladmiral, "Critique \& critiques ", in Dominique Château (dir.), À propos de la critique, Paris, L'Harmattan, 1995 , p. 17
37. D. Château, Préface, in D. Château, J.-R. Ladmiral (dir.), Critique et théorie, Paris, L'Harmattan, 1996, p. 9.
Une tentative de critique « avec les moyens spécifiques de l'architecture " : Bruno Zevi, « L'opera architettonica di Michelangiolo nel quarto centenario della morte. Modelli, fotografie e commenti degli studenti dell'Istituto di Architettura di Venezia ", L'Architettura. Cronache e storia, janvier 1964 pp. 654-712. 
d'individus vis-à-vis des productions humaines, principalement artistiques ${ }^{38}$. „ Au sein de cette deuxième acception (la critique esthétique), on peut à nouveau distinguer deux niveaux, celui des textes d'auteurs « au plus près de I'art », c'est-à-dire, schématiquement, les théories esthétiques, et celui des « réflexions induites directement de I'art, comme terrain d'une investigation intellectuelle ou d'une pratique réelle ${ }^{39}$ ", c'est-à-dire la critique comme pratique de l'évaluation ou du jugement des œuvres. Enfin, notamment aux États-Unis, le mot critique recouvre parfois la théorie critique de l'École de Francfort, ce qui a des retombées importantes dans le domaine des théories de l'architecture depuis le début des années 1970. La théorie critique et ses développements dans le poststructuralisme ont en effet formé la base d'une importante production théorique sur l'architecture, dominante dans les universités américaines.

\section{Un discours interne au champ architectural?}

Quelles significations ces différentes conceptions endossent-elles lorsqu'elles sont transférées à l'architecture? En convoquant l'étymologie, plusieurs théoriciens ou historiens de l'architecture ont proposé des définitions de la critique architecturale. Ainsi, nombre d'écrits théoriques sur l'architecture ${ }^{40}$-à la suite des historiens d'art et des spécialistes de l'esthétique - se réfèrent au sens premier de krinein ${ }^{41}$, "séparer le vrai du faux ", ce qui renvoie au mot krisis, et associe de la sorte la critique à la notion de crise, moment de refondation disciplinaire et moment de refondation des discours. "La perception de la crise constitue le point de départ de la critique ${ }^{42}$. " En 1959, dans Le règne de la critique, Reinhart Koselleck ${ }^{43}$ montrait l'origine commune de crise et de critique: « Le mot "critique" (all. Kritik, angl. criticks, de nos jours seulement criticism) a, avec crise (all. Krise, angl. Crisis), une origine commune dans le grec krinein : distinguer, choisir, juger, décider, trancher [...]. Les Grecs utilisaient krinein et krisis dans une large mesure, même si ce n'était pas l'origine, dans la juridiction et les tribunaux. Crise signifiait d'abord action de distinguer et dispute, mais aussi décision au sens d'un jugement définitif ou d'un jugement tout court, ce qui est aujourd'hui du domaine de la critique. »

Et en effet, dans le champ des théories de l'architecture, l'objet « critique » semble davantage exploré en partant des concepts philosophiques du mot critique, plutôt qu'en se référant à la critique esthétique. Il peut être compris comme une forme de discours spéculatif sur l'architecture, une contribution aux corpus de textes théoriques et non comme un écrit se référant à un édifice et un projet en particulier. Cette acception du mot critique est dominante aux États-Unis. OutreAtlantique, on peut établir une nette distinction entre criticism, entendue comme critique dans la sphère publique ou dans l'espace public médiatique (public criticism, qui inclut la critique dans les grands quotidiens ${ }^{44}$ ), et criticism, comme activité théorique, interne au monde académique et professionnel, ces deux conceptions étant séparées par un fossé grandissant depuis les années 1970. De même, depuis cette date s'est dessinée une nette séparation entre les périodiques professionnels et les revues liées aux départements de théorie et d'histoire de l'architecture des universités américaines.
38. Ibid., p. 10

39. Ibid.

40. Dans ce même dossier, l'article de Manuel Martín Hernández repart des significations du grec krinein.

41. J.-R. Ladmiral, art. cit. note 36, p. 17.

42 , I. de Solà-Morales, op. cit. note 24, p. 5.
43. Reinhart Koselleck, Le règne de la critique, Paris, Les Éditions de Minuit, coll. "Arguments », 1979 (édition originale Kritik und Krise, Fribourg, Karl Alber, 1959).

44. Voir S. Stephens, «Assessing the State of Architectural Criticism in Today's Press ", Architectural Record, vol. CLXXXVI, mars 1998, pp. 64-69. 
Cette distance est bien plus grande qu'en Europe, où a longtemps prévalu une tradition de revues professionnelles et critiques. Encouragée depuis les années 1970 par l'importance, dans le milieu académique américain, des revues Oppositions (1973-1984), Assemblage (19862000), October (1976-), ainsi que ANY (1993-2000), la critique s'est progressivement défaite de la fonction d'évaluation, de jugement de l'architecture contemporaine pour rejoindre un discours théorique relativement autonome par rapport à la production et à la discipline architecturales. Dans une première phase, Oppositions a tenté de construire une forme d'histoire critique, fortement inspirée par les échanges intenses de cette revue avec Tafuri et l'École de Venise, et de redéfinir les rapports entre critique, théorie et histoire, en " enracinant la critique dans I'histoire ${ }^{45}$ ». Or, la place de l'histoire critique défendue un temps dans Oppositions s'est amenuisée au profit d'essais théoriques qui expérimentent les analogies et les transferts de notions entre I'architecture et d'autres champs culturels et disciplinaires ${ }^{46}$ - soit l'affaiblissement de l'exploration diachronique au profit de la recherche de liens synchroniques. Cette tendance s'est poursuivie dans Assemblage, dont le programme post-structuraliste entérine, selon Mitchell Schwarzer, I'« abandon de l'idée de la nécessité d'un lien entre la pratique et la réflexion historique et théorique». Le renoncement - volontaire - à ce lien n'estil pas synonyme de renoncement à la critique, si l'on conçoit cette dernière comme un intermédiaire entre la pratique de l'architecture et un discours théorique? Rompant avec la narration historique et la description propres au commentaire critique, ces deux revues ont assimilé l'architecture à une production textuelle. L'écriture gagne en autonomie par rapport à la discipline architecturale: "La représentation de l'architecture dans le texte était conçue comme une production textuelle $d^{\prime}$ architecture ${ }^{47}$. »

Jane Rendell et lan Borden définissent quant à eux la théorie critique sans la limiter à l'École de Francfort, d'Adorno à Marcuse. Ils en envisagent une conception étendue et actualisée " qui renferme toute théorie émancipatrice et auto-réflexive (divers féminismes, marxismes, psychanalyses, etc.) dans des champs disciplinaires constitués théoriquement (comme les études historiques, les cultural studies, l'anthropologie, la géographie, la sociologie, etc. $)^{48}$. " Le but de la critical theory ainsi étendue aux études féministes, post-coloniales, et appliquée à la critique architecturale est de fonder une histoire critique de l'architecture (critical theorised history), déterminée par sa méthodologie plus que par son recul temporel avec les objets: "De telles histoires de l'architecture peuvent être des histoires du présent aussi bien que du passé ${ }^{49}$."

Dernière déclinaison du mot "critique », le concept de critical architecture se réfère à la capacité critique de I'architecture en elle-même et sur elle-même. Pour K. Michael Hays (Critical Architecture : between Culture and Form, 1984), la capacité critique de l'architecture ne s'adresse pas uniquement aux contextes social, idéologique, économique, biographique ou technologique de I'époque et de l'œuvre ${ }^{50}$ - mais bien à l'architecture comme production intellectuelle autonome, apte à faire avancer la connaissance sur l'architecture elle-même. Hays définit le concept de critical architecture comme
45. Mitchell Schwarzer, " History and Theory in Architectural Periodicals », The Journal of the Society of Architectural Historians (numéro spécial, «Architectural History 1999/2000 »), vol. LVIII, n³, septembre 1999, p. 345.

46. Ibid., pp. $342-348$

47. "Écrire est devenu de plus en plus un moyen pour faire des architectures originales sans tenir compte de la discipline architecturale », ibid., p. 346.
48. Ian Borden, Jane Rendell, « From Chamber to Transformer: Epistemological Challenges and Tendencies in the Intersection of Architectural Histories and Critical Theories ", in I. Borden, J. Rendell, InterSections. Architectural Histories and Critical Theories, Londres, New York, Routledge, 2000, p. 7.

49. Ibid., p. 9
50. Comme le Mouvement moderne à l'égard des conditions sociales et politiques de son époque: voir Hilde Heynen, Architecture and Modernity: a Critique, Cambridge (Mass.), The MIT Press, 1999.

51. K. Michael Hays, "Critical Architecture: between Culture and Form », Perspecta, $n^{\circ} 21$, p. 15.

52. J. Rendell et al., op. cit. note 26 . 
trouvant sa place « entre la représentation efficace de valeurs culturelles préexistantes et l'autonomie totalement détachée d'un système formel abstrait ${ }^{51}$ ». Enfin, des contributions plus récentes définissent la notion de critical architecture comme " quelque chose entre la conception architecturale et la critique ${ }^{52}$ ».

Depuis 2002, la « criticalité » ou la potentialité critique de l'architecture donne en effet lieu à un âpre débat aux États-Unis. Exemplaire de cette discussion, I'article de Robert Somol et Sarah Whiting ${ }^{53}$ conteste la dimension critique et théorique de l'architecture et lui préfère l'idée d'« architecture projective », concernée par l'engagement écologique, social, économique, et issue du pragmatisme. Cet antagonisme a pu être considéré comme un conflit « œdipien » de générations par Reinhold Martin ${ }^{54}$ : schématiquement, une nouvelle génération (représentative de ce moment " post critical ») se dressait contre celle d'Oppositions et contre celle, plus récente, d'Assemblage, qui avaient surplombé le débat théorique américain pendant trente ans ${ }^{55}$. Plusieurs auteurs ont souligné que durant trois décennies, la domination de la critical architecture et la croyance dans la potentialité critique d'une architecture « réflexive ou auto-réflexive » a relégué la critique écrite - au sens de Baudelaire et de T. S. Eliot ${ }^{56}$ à l'arrière-plan. Elle aurait même contribué à son effacement progressif ${ }^{57}$.

Si cette analyse n'épuise pas l'ensemble des acceptions du mot critique en relation avec les théories architecturales, elle montre que dans les dernières significations évoquées, la critique perd sa spécificité de jugement - effacement renforcé par la domination théorique de la critical architecture.

\section{La critique architecturale, une forme de critique} " en général »? Liens avec la critique d'art

Des critiques d'architecture ont souligné les possibles liens entre critique littéraire (" source et moule de toutes les autres formes de critique », selon Yorgos Simeoforidis ${ }^{58}$ ) et critique architecturale ${ }^{59}$. La perméabilité des théories architecturales à la linguistique, à la sémiotique et aux théories littéraires (plus qu'à la critique littéraire ellemême) dans les années 1960 et 1970, n'est pas étrangère à un tel rapprochement ${ }^{60}$. Historiens et critiques d'architecture sont moins enclins à établir des parallèles avec la critique d'art. Bien que répandue parmi les architectes, cette position semble discutable, du moins d'un point de vue méthodologique. Le refus de l'analogie avec la critique d'art part notamment de l'hypothèse que depuis le XIXe siècle le lien entre critique architecturale et critique d'art aurait été rompu au moment où la critique architecturale n'a plus pu se fonder sur l'étude " psychologique » et « biographique » des auteurs et des œuvres - vision, notons-le, pour le moins anachronique de la critique d'art. Le rejet d'une possible analogie avec la critique d'art s'appuie aussi sur un truisme: I'architecture ne peut se réduire aux arts visuels, étant donné les multiples cadres (esthétique, technique, social, économique) dans lesquels elle est inscrite.

Partir de la critique d'art pour mieux cerner la critique architecturale et pour la construire en objet de recherche peut donc paraître un parti pris, qui implicitement affirmerait une dépendance ou un lien de la critique architecturale vis-à-vis à la critique d'art. Or, on peut tirer profit des avancées épistémologiques de l'histoire de la critique d'art, qui depuis le début des années $1980^{61}$ a
53. "Notes around the Doppler Effect and Other Moods of Modernism », Perspecta, The Yale Architectural Journal, n॰33, 2002, pp. 72-77. Voir une interprétation de ce débat dans ce dossier, dans l'article de Manuel Martín-Hernández, «Vers une théorie et une critique de l'architecture ».

54. Reinhold Martin, "Critical of What? Toward a Utopian Realism », Harvard Design Magazine, $n^{\circ}$ 22, printemps/été 2005, pp. 104-109.
55. R. Martin (art. cit. note 54) épingle l'interprétation de Somol et Whiting comme une lecture déformante de la philosophie de Gilles Deleuze et de Félix Guattari, qui ont fortement imprégné la théorie architecturale américaine des années 1990 Voir aussi Daniel A. Barber, " Militant Architecture. Distabilising Architecture's Disciplinarity ", in J. Rendell et al., op cit. note 26 , pp. 57-66.
56. Introduction à "The Space of Criticism », Lotus, n० 72, mai 1992.

57. John Macarthur, Naomi Stead, "The Judge is Not an Operator: Historiography Criticality and Architectural Criticism ", Oase, $n^{\circ}$ 69, pp. 116-129.

58. Yorgos Simeoforidis, "Adrift", Lotus, n० 92, mai 1992 («The Space of Criticism »), p. 137-138. 

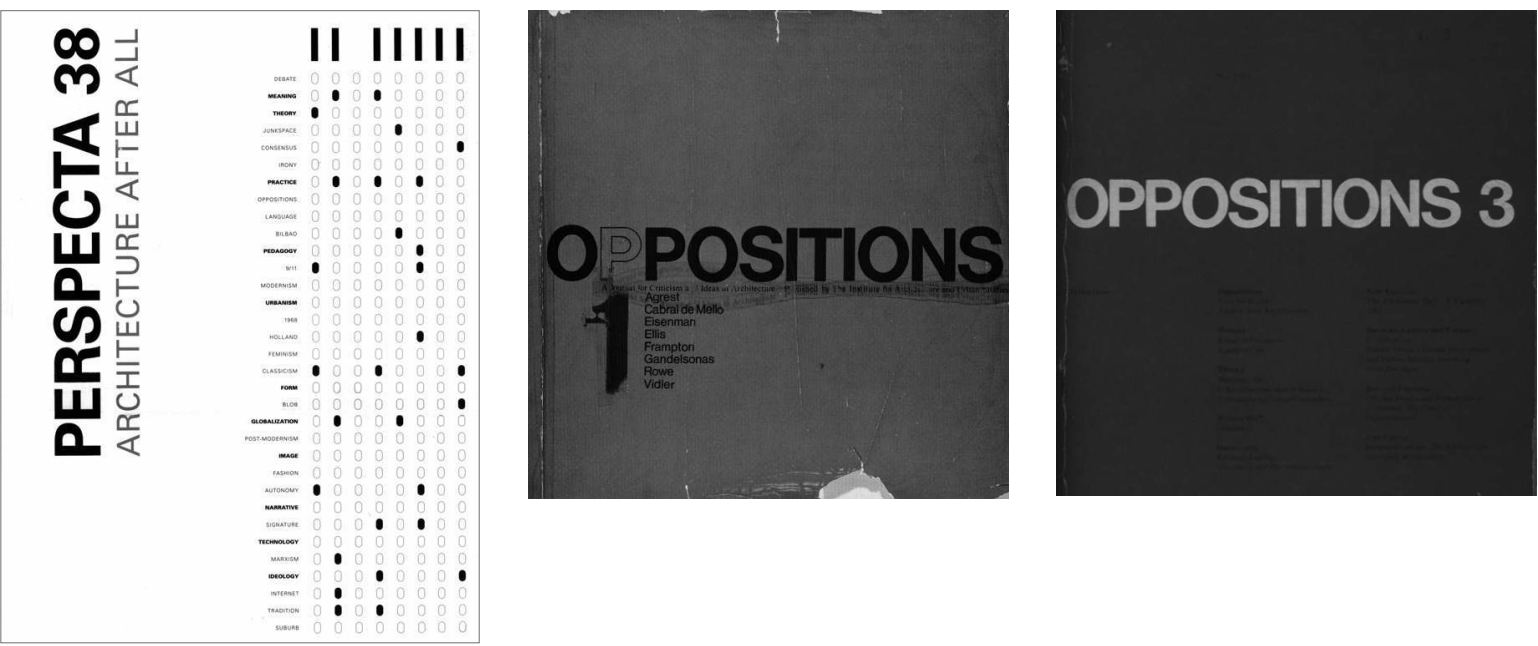

En haut:

Revues théoriques

des années 1970

et 1980 (Assemblage,

Oase, Oppositions,

Perspecta).
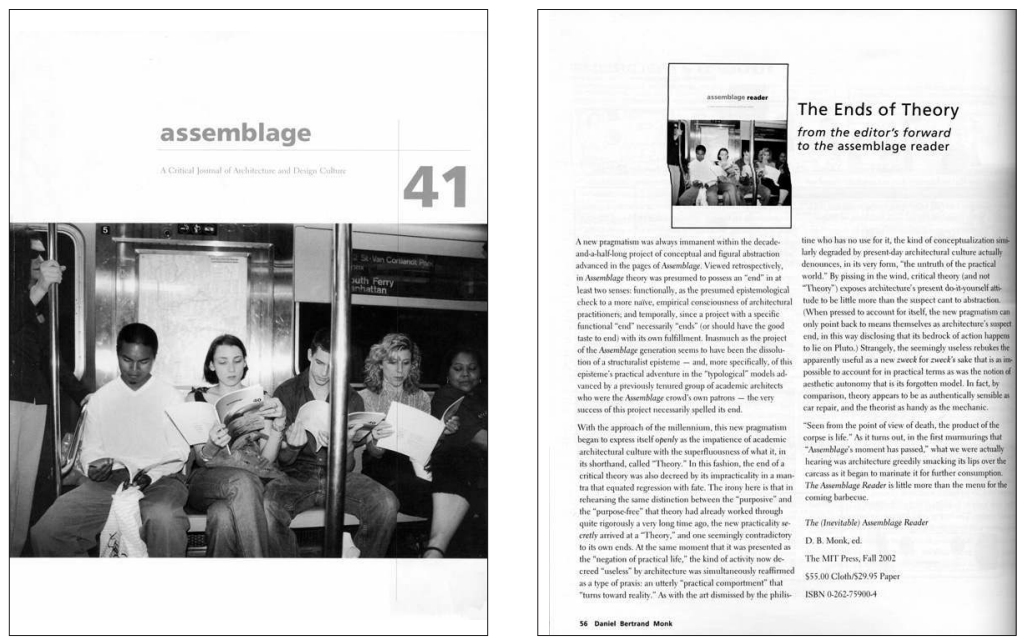

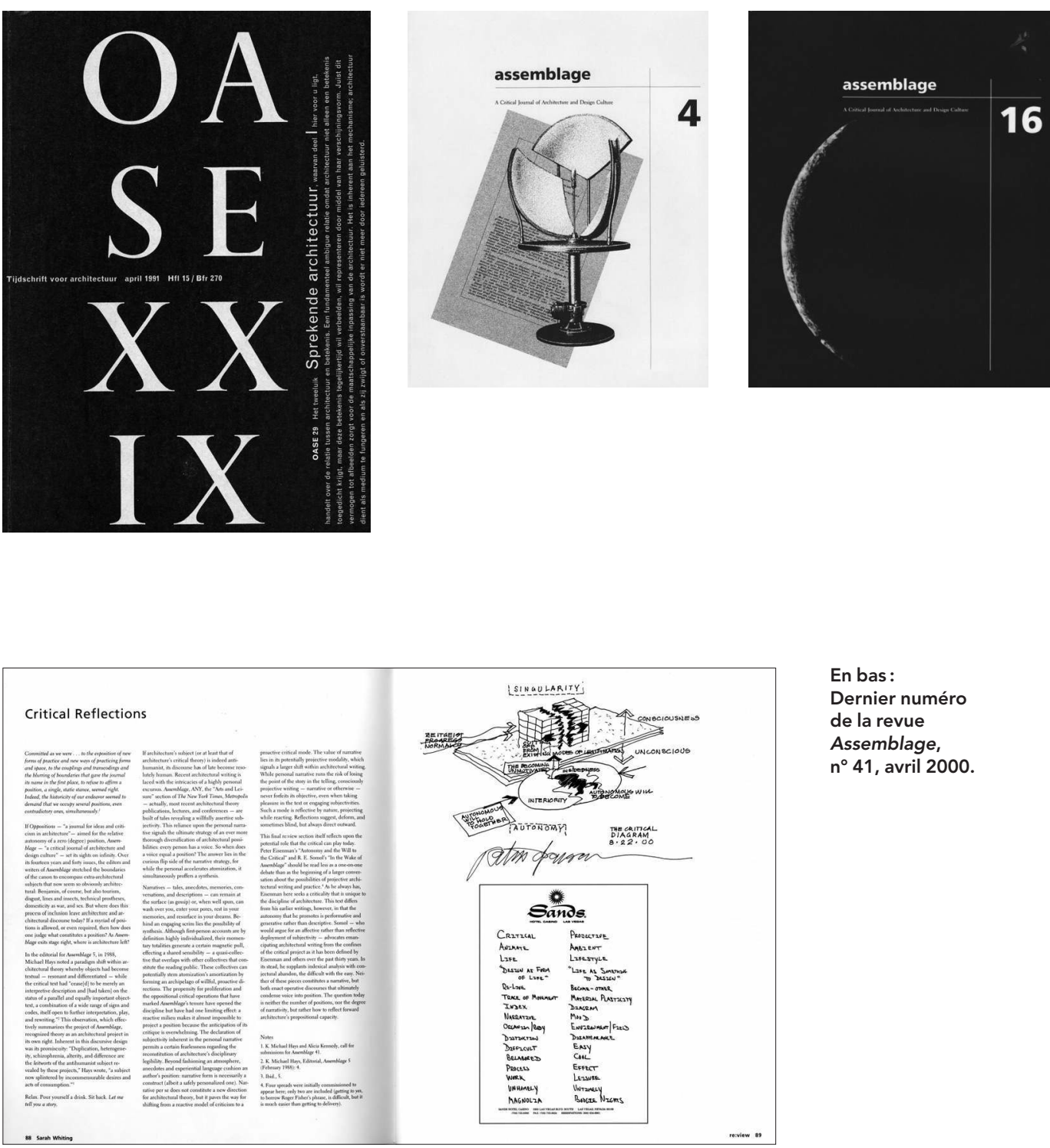

En bas:

Dernier numéro

de la revue

Assemblage,

$n^{\circ} 41$, avril 2000. 
contribué à définir l'objet "critique », à lui conférer la légitimité d'un objet de recherche, à identifier les corpus à la base de son étude et, enfin, à cerner les variations de l'objet « critique » en fonction des points de vue disciplinaires adoptés. L'histoire de l'art propose ainsi plusieurs acceptions du mot critique qui peuvent aussi aider à éclairer les différents registres de critique architecturale. Revenons sur la première signification de « critique architecturale », commentaire critique qui évalue et interprète l'œuvre dans sa singularité.

Cette conception est issue de la définition historique de " critique " au sens de " jugement de goût », initialement formulée en 1915 par I'historien d'art allemand Albert Dresdner, comme " genre littéraire autonome qui a pour objet d'examiner l'art contemporain, d'en apprécier la valeur et d'en influencer le cours ${ }^{62} »$. Elle provient de la montée en puissance de la critique d'art au XVIII e siècle, en liaison avec l'organisation d'expositions publiques et, principalement, des Salons: La Font de Saint Yenne en est le premier réel représentant, et Diderot l'emblème. Depuis cette définition canonique de la critique d'art - " genre littéraire » issu d'une pratique, celle du " critique écrivain » aux XVIII et XIXe siècles (objet d'étude de l'histoire littéraire) ou, du point de vue des sociologues, intermédiaire dans un marché de l'art transformé au XIX siècle par la montée en puissance des expositions personnelles (" agents de socialisation de l'art "63", les critiques alors "font et défont " les carrières des artistes) -, le rôle de la critique d'art dans le débat public comme dans la sphère artistique s'est radicalement modifié. Les critiques ont remis en cause la fonction normative de la critique, notamment lors de la montée en puissance de l'art conceptuel et du minimalisme, puis ont développé de nouveaux instruments d'analyse issus du structuralisme puis du post-structuralisme ${ }^{64}$. Malgré ces transformations radicales, les historiens d'art qualifient, aujourd'hui encore, la critique par deux spécificités: sa fonction d'évaluation qui traverse et fédère d'autres fonctions (interprétation, expression) du discours, et le fait de " parler des œuvres d'art non en général mais dans leur singularité même ${ }^{65}$ ». Transposer cette définition à la critique architecturale, perçue alors comme une pratique indexée sur le présent, implique de considérer que l'histoire de la critique architecturale commence soit avec les commentaires du Salon, lorsque l'architecture y est exposée ${ }^{66}$, soit avec l'essor des revues professionnelles, au milieu du XIX ${ }^{e}$ siècle. Aujourd'hui, elle implique une conception de la critique référée à des projets et des édifices et pouvant participer du débat public. Tout en gardant son autonomie par rapport à d'autres types de discours sur l'architecture, elle ne se coupe pas pour autant de ses relations avec l'histoire et avec les théories architecturales - elle serait, selon les termes de Josep María Montaner ${ }^{67}$, un terrain de « vérification » des théories.

Beaucoup plus large, la seconde signification de " critique d'art » ne s'attache pas à une pratique sociale. En recoupant étroitement le domaine des théories et celui de l'histoire, elle s'inscrit dans le sillage de la position néo-idéaliste de I'historien d'art Lionello Venturi (1885-1961) ${ }^{68}$, lui-même fortement influencé par la philosophie de Benedetto Croce (1866-1952) ${ }^{69}$. Ouvrage pionnier, History of Art Criticism (1936) de Venturi considère l'histoire de la critique comme l'histoire des jugements portés sur
59. Voir Jacques Lucan, « Jusqu'où la critique doit-elle exacerber l'individualisme des architectes? " Le Visiteur, $n^{\circ} 1$, automne 1995, pp. 108-199. Voir également l'article de J. Lucan dans ce dossier, qui traite de la question du langage.

60. L. Martin, thèse citée note 11, p. 15. 61. Hormis quelques écrits pionniers de la fin du XIXe siècle, les études sur la critique d'art ont été entreprises au milieu des années 1960, d'abord en histoire littéraire (qui se restreignait aux écrits critiques des écrivains et poètes) puis en sociologie de l'art (à partir du livre de Cynthia et Harrison White, Canvases and Careers: Institutional Change in the French Painting World, 1965, trad. La carrière des peintres au XIX siècle, Paris, Flammarion, 1991) et, finalement, en esthétique et en histoire de l'art.

62. Albert Dresdner, Die Entstehung der Kunstkritik in Zusammenhang der
Geschichte des europaïschen Kunstlebens, Munich, Bruckmann, 1915, p. 11; trad. La genèse de la critique d'art dans le contexte historique de la vie culturelle européenne, Paris, École nationale supérieure des beaux-arts, 2005, p. 31.

63. C. Amey, op. cit. note 35, p. 15.

64. Sur la critique des années 1960 et 1970 aux États-Unis, voir Hal Foster, "Art Critics in Extremis », in Design and Crime (and 


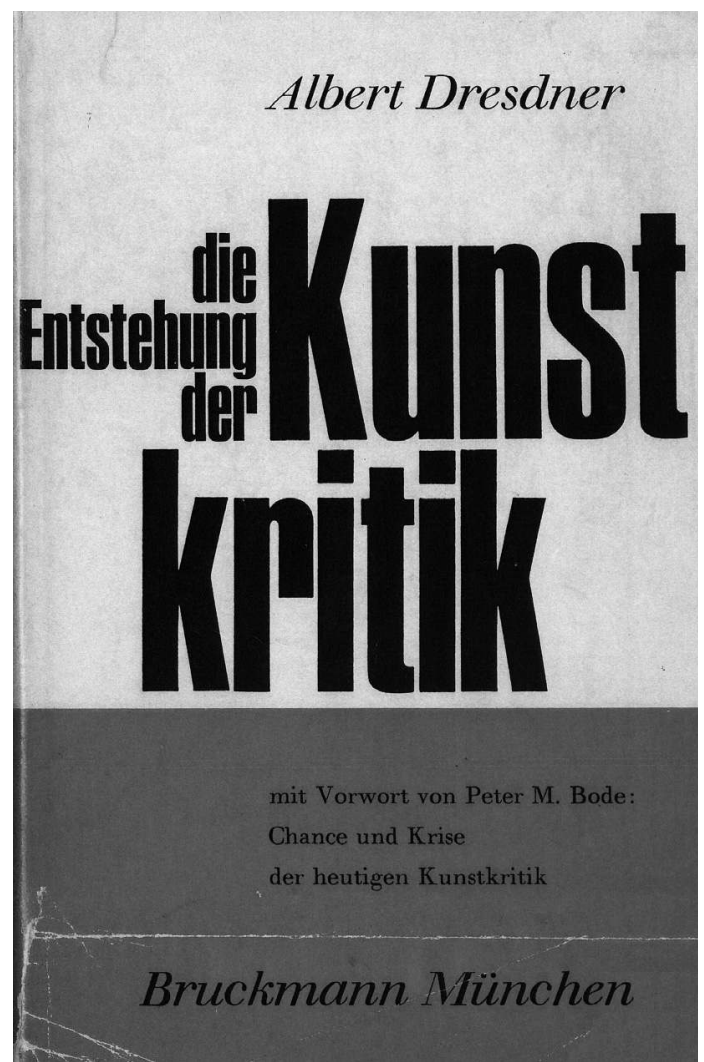

Albert Dresdner,

Die Entstehung

der Kunstkritik,

Munich, Bruckmann,

1915 ; édition

de 1968.

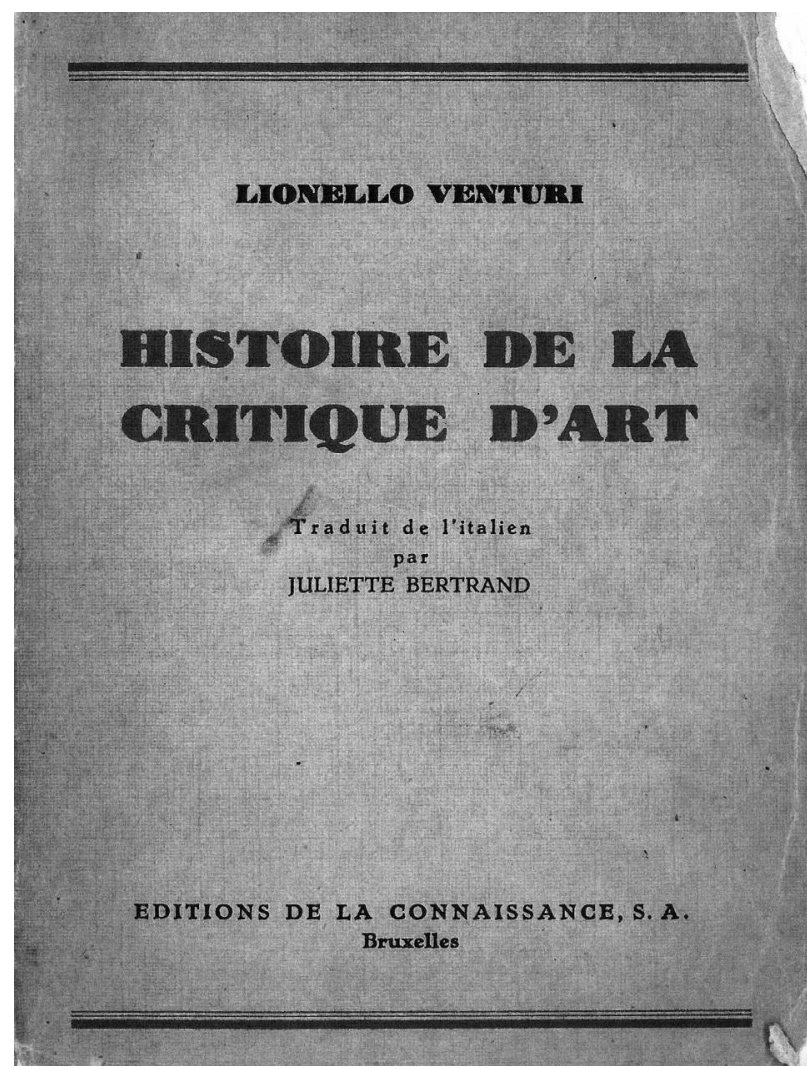

Lionello Venturi,

Histoire de la critique

d'art, Bruxelles,

Éditions de la

Connaissance, 1938. other Diatribes), Londres, New York, Verso, 2002, pp. 104-122; Id., Le retour du réel: situation actuelle de l'avant-garde, Bruxelles, La Lettre volée, 2005 [édition originale

The Return of the Real, The MIT Press, 1996].

65. Jean-Marc Poinsot, Pierre-Henry Frangne, "Histoire de l'art et critique d'art. Pour une histoire critique de l'art », in J.-M. Poinsot, P.-H. Frangne (dir.), L'invention de la critique d'art, Rennes, Presses Universitaires de Rennes, 2002, p. 9.
66. Emmanuel Schuck, « La critique et la section d'architecture dans les salons de la seconde moitié du XIX siècle », Romantisme. Revue du dix-neuvième siècle, n 71

$1^{\text {er }}$ trimestre 1991, "Critique et art », pp. 49-56.

67. Josep María Montaner, Arquitectura y critica, Barcelone, Gustavo Gili, 1999; traduction française partielle: "Matière et technique de la critique », in A. Deboulet et al., op. cit. note 6, pp. 123-136.
68. Lionello Venturi, History of Art Criticism, New York, E. P. Dutton and Co, Inc., 1936. Le livre est ensuite édité pour la première fois en français en 1938 (Bruxelles, Éditions de la Connaissance), puis publié pour la première fois en Italie en 1945. Dans ce qui suit, nous nous référons à l'édition française de 1969 (Flammarion), traduction de l'édition italienne (Giulio Einaudi) de 1964

69. Gianni Carlo Sciolla, La critica d'arte del Novecento, Turin, Utet, 1995, pp. 3-35. 


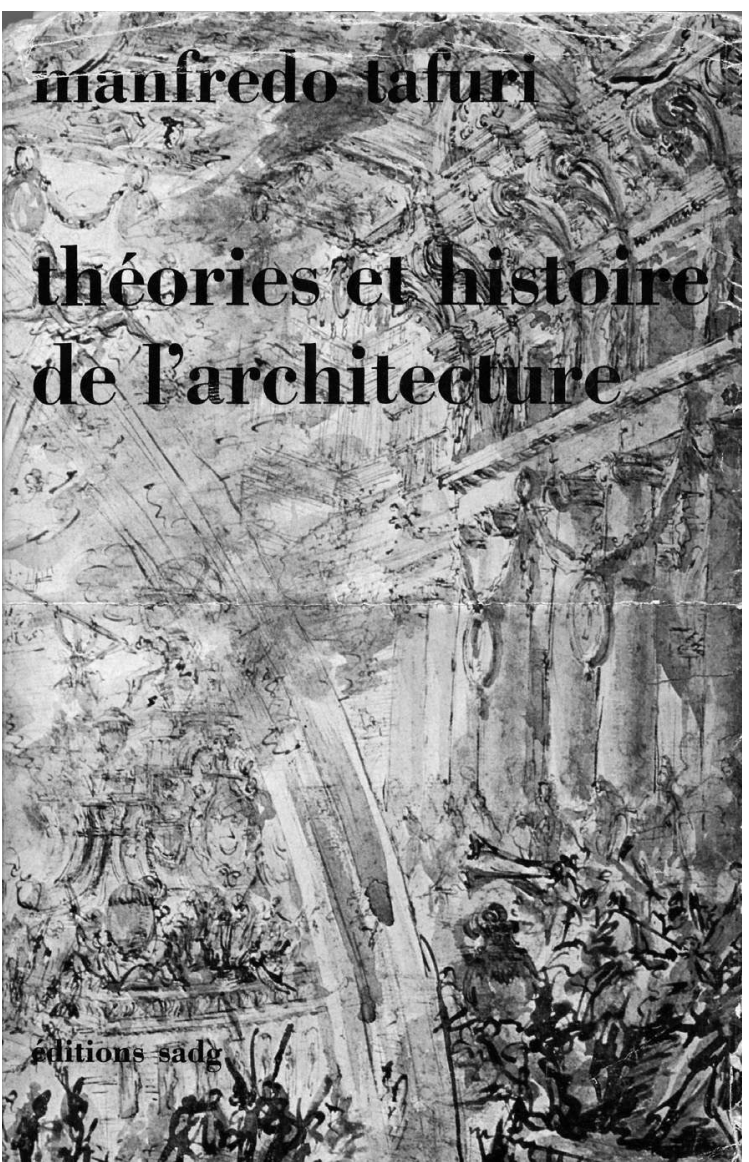

Manfredo Tafuri,

Théories et histoire

de l'architecture,

Paris, SADG, 1976

[édition originale 1968]. l'art, donc la critique comme l'ensemble des théories artistiques qui permettent de juger l'art. La critique comprend de la sorte la pensée critique sur l'art, l'histoire de l'art à la condition qu'elle comporte un aspect critique, sans lequel elle n'est qu'une accumulation positiviste de faits. Allant à l'encontre de l'idée de la critique comme genre littéraire, Venturi écrit que cette conception d'origine française accorde " une trop grande importance au côté pratique et social de la critique au détriment des jugements portés sur les œuvres $d^{\prime} \operatorname{art}^{70}{ }^{\prime \prime}$.

Impliqué dans le débat architectural italien de l'entredeux-guerres, Venturi a eu une influence considérable sur la critique d'architecture italienne de l'entre-deuxguerres puis de l'après-guerre, imprégnée elle aussi de la philosophie crocéenne. À la mort de Croce en 1952, Bruno Zevi (1918-2000) a en effet remis à I'honneur le texte de ce philosophe sur la méthode historique en architecture ${ }^{71}$, qu'il considère être à la base d'une méthode d'histoire critique de l'architecture. Malgré la remise en question, dès les années 1950, des thèses de Venturi sur la critique d'art, et bien que la conception de Venturi, à la suite de Croce, de l'architecture comme forme d'art (la critique architecturale ne traitant à ses yeux que de l'aspect esthétique, séparé du versant utilitaire de la construction) ait également été rapidement battue en brèche, en Italie la proximité entre histoire et critique est restée dominante dans le domaine de I'architecture.

On se rappelle l'injonction, probablement polémique, de Manfredo Tafuri, affirmant qu'il n'y a pas de critique, mais uniquement de l'histoire ${ }^{72}$ : il faut comprendre qu'il n'y a pas de place pour la critique en dehors d'une analyse
70. L. Venturi, Histoire de la critique d'art, Paris, Flammarion, 1969, p. 31.

71. Bruno Zevi, «Benedetto Croce e la riforma della storiografia architettonica », Metron, n47, 1952.
72. "There is no criticism, only history: interview with Manfredo Tafuri by Richard Ingersoll », Casabella, n 619-620, janvier-février 1995, pp. 96-101. 
historique. Or, les significations de la critique et ses rapports avec l'histoire ont été explorés de manière plus subtile, au-delà de l'identification venturienne entre histoire et critique, par Tafuri-notamment, dans Théories et histoire de l'architecture et plus tard, dans « II progetto storico ", introduction à La Sfera e il Labirinto. Le substrat historique de la critique est invoqué : critiquer, c'est pour Tafuri porter un jugement de valeur non sur l'œuvre in abstracto, mais sur sa pertinence à un moment historique donné.

\section{Des pistes à explorer}

Au-delà des différentes significations ici déclinées, on peut in fine distinguer deux points de vue sur la critique architecturale, qui en grande partie façonnent sa construction en objet de recherche. Le premier la considère comme un discours interne (acteurs, références théoriques, supports de presse) au champ de l'architecture. C'est ce que souhaitait Peter Collins lorsqu'il écrivait: " $C^{\prime}$ est une activité que l'on doit considérer "sui generis" [...] activité tout à fait particulière, et exclusivement liée à l'architecture ${ }^{73}$. " Dans Architectural Judgement (1971), Collins va jusqu'à affirmer que les procédures judiciaires et le diagnostic médical (deux activités qui, notons-le, faisaient appel aux significations anciennes du mot critique, trancher et « peser le pour le contre ${ }^{74}$ ) sont plus fondés que la critique d'art à être rapprochés de la critique d'architecture ${ }^{75}$. Au séminaire de Cranbrook en 1964, il expose que la critique architecturale doit être un discours professionnel spécialisé qui prenne en compte non l'« œuvre », comme le fait traditionnellement la critique d'art, mais le processus de conception : dans un but d' " efficacité », la critique devrait s'appliquer aux esquisses préliminaires plutôt qu'à l'édifice achevé. Cela tend à affirmer l'autonomie disciplinaire de la critique architecturale, en relation exclusive avec l'histoire et les théories de l'architecture. Qu'en estil alors des instruments de la critique, lorsque ceux-ci proviennent d'autres champs disciplinaires (notamment littérature, art, sciences humaines et sociales) et sont transférés à l'analyse de l'architecture, comme ce fut souvent le cas dans la critique de la seconde moitié du XXe siècle? Le second point de vue perçoit à l'inverse la critique architecturale comme l'une des « formes de la critique en général ». Adopter cette optique n'implique pas de penser l'architecture comme une forme d'art dans une posture néo-idéaliste. Considérer la critique architecturale comme une forme de la critique en général permet de la différencier d'autres types de discours sur I'architecture: ce n'est plus l'autonomie du discours architectural mais les spécificités (auteurs, registres, rhétoriques) d'une forme de discours qui sont alors au centre de la recherche.

Cette opposition entre d'un côté, une critique « autonome », interne à la discipline architecturale, et, de l'autre, une critique aux prises avec la critique d'art et ses fonctions sociales est à rapprocher des relations de la critique à l'espace public. Un des aspects fondamentaux de la critique est en effet son interaction (ou non) avec un espace public de débat, interaction au cœur même de la définition de la critique. Car la critique d'art historiquement définie comme " genre littéraire » et comme « jugement " a partie liée avec l'émergence, au XVIII siècle, d'un espace public de discussion. Cette intersection avec

73. P. Collins, op. cit. note 5, p. 5.

74. Voir R. Koselleck, op. cit. note 43, pp. 164-166.

75. P. Collins, op. cit. note 7, p. 142. 
la sphère publique ne tend-elle pas à disparaître lorsque la critique est entendue comme discours disciplinaire et qu'elle se proclame pratique « autonome »?

Considérer la critique architecturale comme forme d'une " activité plus large appelée critique » permet enfin de tirer profit des avancées dans plusieurs domaines de recherche connexes, parmi lesquels les recherches sur la critique d'art. Avant même l'histoire de l'art, la sociologie de l'art et l'histoire sociale de l'art ont contribué à définir la critique, ses fonctions dans le marché et dans l'espace public de discussion, son influence, notamment au $X I X^{e}$ siècle, sur la carrière des peintres. Les fonctions sociales de la critique architecturale ont commencé d'être partiellement explorées, en relation notamment avec I'histoire des professions et avec la recherche sur les conséquences de la publication en architecture ${ }^{76}$. L'histoire de l'art a ensuite renouvelé les corpus d'étude et ainsi contribué à redéfinir l'objet « critique »: par rapport aux premières études, menées par les historiens de la littérature, I'histoire de l'art a progressivement pris en compte des corpus étendus à la critique dite ordinaire, au-delà des seuls écrits d'écrivains, d'historiens ou de critiques dont l'aura a été préalablement construite dans les champs scientifique ou littéraire ${ }^{77}$.

Une réflexion comparable pourrait être menée pour la critique architecturale et les corpus qui contribuent à la délimiter. Son histoire n'est en effet pas seulement celle des "grands critiques", reconnus par ailleurs comme historiens ou théoriciens de l'architecture. Pour la contribution - certes fort importante - de ces derniers, I'histoire de la critique peut s'appuyer sur les biographies intellectuelles que l'« histoire de l'histoire de l'architecture » a progressivement éclairées, s'intéressant à des historienscritiques comme Pevsner, Giedion, Hitchcock, Behrendt ou Banham pour I'historiographie du Mouvement moderne, s'étendant actuellement à des critiques ou historiens moins impliqués dans cette critique « opératoire ». Une autre piste de recherche concerne les interactions entre revues professionnelles, presse généraliste, culturelle et critique architecturale, qui méritent d'être analysées avec attention. Enfin, les travaux sur la critique entrent également en résonance avec les récentes études sur la réception.

Sauf peut-être à opérer dans le champ de l'esthétique, de la sociologie de l'art ou de la sociologie des professions, on ne peut, en effet, définir « la » critique architecturale comme une pratique ou un type de discours uniques. L'identification d'un objet de recherche devra nécessairement se référer à des conjonctures particulières, qui ont façonné des configurations de la critique à des moments historiques et dans des milieux culturels déterminés. Ainsi, l'objet « critique » pourra être abordé à l'aide de plusieurs paramètres, plus aisément définissables: les acteurs de la critique, ses instruments théoriques, dans des contextes historiques et épistémologiques déterminés. C'est ce qu'a tenté d'esquisser ce dossier des Cahiers de la recherche architecturale et urbaine en proposant plusieurs essais sur la critique, historiquement et culturellement situés.

76. Voir les travaux d'Hélène Lipstadt et ceux de Beatriz Colomina.

77. Voir Jean-Paul Bouillon, "La critique d'art dans la seconde moitié du XIX siècle : nouvel aperçu des problèmes », Quarantehuit / Quatorze, n 5, $1^{\text {er }}$ trimestre 1993, p. 32. 